\title{
Educação em saúde e promoção da saúde: impacto na qualidade de vida do idoso
}

\author{
Health education and health promotion: impact on quality of life of \\ elderly
}

Janaina Pinto Janini ${ }^{\mathbf{1}}$, Danielle Bessler ${ }^{\mathbf{2}}$, Alessandra Barreto de Vargas ${ }^{\mathbf{3}}$

RESUMO Objetiva-se analisar o impacto das ações de promoção e educação em saúde na busca da qualidade de vida, na autonomia e no autocuidado da pessoa idosa. A coleta de dados deu-se por meio de entrevistas semiestruturadas com 83 idosos, de ambos os sexos, e evidenciaram a importância da intersetorialidade e da realização de ações educativas emancipadoras da população. Ainda assim, faz-se necessário agregar, a esses processos, estratégias de universalização e equalização das informações concedidas aos idosos. Concluiu-se que a educação constitui uma poderosa ferramenta na promoção da saúde, na garantia da autonomia e na qualidade de vida do idoso.

PALAVRAS-CHAVE Promoção da saúde; Educação em saúde; Autonomia pessoal; Qualidade de vida; Idoso.

ABSTRACT It aims to analyze the impact of the actions of promotion and health education in the pursuit of quality of life, in the autonomy and in the self-care for the elderly person. The data collection took place through semi-structured interviews with 83 seniors, of both genders, and emphasized the importance of inter-sector and the realization of emancipatory educational activities of the population. However, it is necessary to add to these processes, strategies of universalization and equalization of the information provided to elderly people. It is concluded that education is a powerful tool in promoting health, in ensuring the autonomy and in quality of life of the elderly.

KEYWORD Health promotion; Health education; Personal autonomy; Quality of life; Elderly.

\footnotetext{
1 Universidade Gama Filho - Rio de Janeiro (RJ), Brasil. janapjanini@yahoo.com.br

2 Fundação Oswaldo Cruz (Fiocruz), Escola Politécnica de Saúde Joaquim Venâncio (EPSJV) - Rio de Janeiro (RJ), Brasil. danibessler@hotmail.com 


\section{Introdução}

A promoção da saúde é uma estratégia que proporciona visibilidade aos fatores de risco e aos agravos à saúde da população, focando no atendimento do indivíduo (coletivo e ambiente) e elaborando mecanismos que reduzem as situações de vulnerabilidade (BUss, 2000). As origens e concepções da promoção da saúde tiveram início com o advento da educação em saúde, no início do século XX, a partir da observação da alteração dos índices de adoecimento decorrentes de práticas educativas realizadas por "higienistas" da época (ENRIA; STAFOLANI, 2010, P. 173). Naquele período, então, o significado da promoção da saúde era atribuído a ações de educação em saúde, visando à melhoria da qualidade de vida. Embora a educação em saúde possua caráter mais amplo, ela é considerada um dos principais dispositivos para a viabilização da promoção da saúde, auxiliando no desenvolvimento da responsabilidade individual e na prevenção de doenças (LOPES; SARAIVA; XIMENES, 2010).

Nesse sentido, a educação possui importância inegável para a promoção da saúde, sendo utilizada como veículo transformador de práticas e comportamentos individuais, e no desenvolvimento da autonomia e da qualidade de vida do usuário (LOPES; SARAIVA; XIMENES, 2010).

Esses conceitos, associados a outras políticas públicas, foram devidamente ajustados às demandas populacionais e inseridos na Política Nacional de Promoção da Saúde (PNPS), cujo objetivo está voltado à promoção da qualidade de vida através da intervenção "em diversos segmentos sociais condicionantes de saúde, como trabalho, habitação, ambiente, educação, lazer, cultura, acesso a bens e serviços essenciais" (BRASIL, 2010, P. 17). Tais condicionantes estão inegavelmente relacionados à qualidade de vida, oportunizados através de práticas promocionais e educacionais em saúde.
Todavia, esse caminho é menos percorrido quando nos referimos à longevidade. Apesar de a PNPS possuir o incentivo à pesquisa como diretriz (BRASIL, 2010), ainda são necessários estudos que vislumbrem as atuais demandas básicas do longevo, a metodologia aplicada pelos profissionais de saúde para o alcance dos objetivos do programa e os entraves na sua implantação, bem como as adequações necessárias para a sua viabilização.

O paradigma promocional maximiza a necessidade de estudos no processo de produção do conhecimento e das práticas no campo da saúde, para garantir uma melhor exequibilidade desse modelo. Isto posto, o objetivo deste estudo é avaliar o impacto da inserção dos programas de promoção e educação em saúde na autonomia, na qualidade de vida e no autocuidado do idoso, através de investigação do conhecimento e orientação do mesmo acerca da percepção de mudanças na própria saúde; o estímulo e o desenvolvimento de habilidades pessoais que garantam a autonomia e o autocuidado; a qualidade das práticas educativas e os métodos avaliativos dentro desse processo.

\section{Método}

O estudo caracteriza-se como de abordagem qualitativa, de natureza descritiva e exploratória. As questões éticas foram observadas em todas as etapas da pesquisa, atendendo à Resolução nº 196/96 do Conselho Nacional de Ética, sendo a pesquisa aprovada pelo Comitê de Ética em Pesquisa da Secretaria Municipal de Saúde do Rio de Janeiro.

O cenário da pesquisa foi uma Unidade Básica de Saúde (UBS) localizada na Zona Oeste do Município do Rio de Janeiro, e tem como amostra 83 idosos atendidos na instituição, sendo 48 do sexo feminino e 35 do sexo masculino; todos com faixa etária entre 60 e 83 anos. Os critérios de inclusão no estudo foram idade superior a 60 anos e estar cadastrado na instituição. 
Foi utilizado como instrumento de coleta de dados um roteiro de entrevista semiestruturado, contendo: 1) perfil do usuário; 2) frequência de consultas; 3 ) conhecimento de doenças ou problemas inerentes ou suscetíveis à idade; 4) conhecimento para identificar fatores de risco ou agravos à saúde; 5) prática e orientação sobre hábitos saudáveis e autocuidado com a saúde; 6) participação em atividades educativas: métodos, técnicas e avaliação.

Para a análise dos dados empíricos, utilizou-se a técnica de análise temática. Durante a leitura exaustiva dos discursos, buscando a apreensão das ideias centrais e a estrutura de significados, originaram-se três itens temáticos: perfil etário, gênero e condições de vida; hábitos de vida do idoso; educação, participação social e empoderamento.

A última etapa da análise constituiu-se da interpretação dos dados frente ao referencial teórico específico referente à promoção e à educação em saúde, à vulnerabilidade, ao idoso, à qualidade de vida e ao empoderamento, acrescidos da Política Nacional de Promoção da Saúde e de outros manuais do Ministério da Saúde que se reportam a essa temática.

\section{Resultados e discussão}

Dos 83 idosos entrevistados, 48 (57,83\%) pertencem ao sexo feminino e $35(42,17 \%)$ ao sexo masculino. Estudos apontam a influência do gênero na busca pelos serviços de saúde e indicam uma predominância dessa procura pelo sexo feminino, que pode ser decorrente do imaginário social de que as mulheres teriam prioridade nos estabelecimentos de atenção primária, em relação aos homens. Esse 'status feminino' conferido às UBS pode ser a motivação para a alta demanda de mulheres, bem como a baixa procura dos homens, que objetivam preservar sua "identidade masculina" construída socialmente (FIGUEIREDO, 2005, P. 106). Esse contexto mostra que a qualidade de vida não está relacionada exclusivamente às condições de saúde, e sim à 'preservação' de valores e práticas edificados histórica e culturalmente (MINAYO; HARTZ; BUSS, 2000).

Nesse sentido, as idosas representaram a maior incidência de consultas semestrais: 41 (85,42\%), revelando o cuidado com o corpo através da prevenção, da detecção precoce e/ ou do controle de morbidades. $\mathrm{O}$ fato de as mulheres recorrerem às UBS mais frequentemente pressupõe uma justificativa para a preponderância da longevidade das mesmas em relação aos homens, vislumbrada pelo percentual elevado de viúvas encontrado no grupo entrevistado: 31 (64,58\%). Percebese, no caso das longevas, a associação direta entre qualidade de vida e estado de saúde, muitas vezes definidos na literatura como equivalentes (GUYATT; FEENY; PATRICK APUD SEIDL; ZANNON, 2004, P. 584).

Já a maioria dos homens é casada 32 (91,42\%) e apresenta um número maior de consultas anuais $22(62,86 \%)$. Tal estatística demonstra uma busca mais intervalada e em menor fluxo, se comparada à das mulheres idosas cadastradas na mesma unidade de saúde. Isso pode ser atribuído ao baixo interesse pelo autocuidado atrelado a uma ideia de 'imunidade ao adoecimento'. No entanto, dados sobre a morbimortalidade dos homens idosos desmistificam a ideia de que os mesmos adoecem menos do que as mulheres, mas ainda assim vivem sob tal premissa, assumindo práticas e comportamentos mediante os "significados culturais construídos e atribuídos às diferenças sexuais" (KORIN, 2001, P. 68).

Não se podem descartar as questões de gênero que hierarquizam o sexo biológico e naturalizam um modelo hegemônico de masculinidade, na família e sociedade, pautadas na caracterização de um estereótipo dos homens como "ativos, fortes, capazes do trabalho físico árduo, produtivos, competitivos e orientados ao mundo externo, capazes de combater em guerras e penetrar o 
corpo de uma mulher", sendo inferiorizados quando não apresentam tais requisitos (KORIN, 2001, P. 69). Essa imagem de força seria fragilizada pela menção da preocupação do idoso com sua saúde e com o aumento da demanda espontânea do mesmo no atendimento dentro da atenção primária. Agregados às questões de gênero, podem existir outros fatores interferentes nesta demanda, tais como o cenário organizacional dos serviços e a capacitação dos profissionais de saúde (ALVES ET AL., 2011).

A visão de si mesmo e sua vulnerabilidade não só podem estar associadas à demanda de consultas nas unidades básicas, como também funcionam como motivadoras, ou não, do cuidado com a saúde. A valorização da questão cultural que se reporta ao sexo biológico deve ser levada em consideração nesse processo, pois a sociedade concede ao homem um status de poder capaz de fomentar uma crença de imunidade, ao passo que as mulheres sempre estiveram vinculadas, histórica e culturalmente, à questão do cuidado. Apresenta-se a necessidade de romper com essas explicações pautadas no biológico e superar, "no plano das ações de políticas públicas, as assimetrias conceituais que fomentam percepções equivocadas de vulnerabilidade" (CARRARA ET AL., 2010, P. 19).

Tendo em vista a identificação desse cenário de assimetrias, e na tentativa de reverter as consequências decorrentes da trajetória histórico-cultural atrelada às questões de gênero, o Ministério da Saúde lançou, em agosto de 2009, a Política Nacional de Atenção Integral à Saúde dos Homens (PNAISH), com vista ao estímulo promocional e preventivo da saúde.

Com relação ao conhecimento acerca de doenças ou problemas provenientes do envelhecimento, as mulheres - 47 (97,91\%) - se mostraram mais informadas dos que os homens - 29 (82,85\%). Ambos referiram adquirir tais conhecimentos em atividades em grupo, palestras dentro das unidades de saúde e panfletos distribuídos como métodos educativos aplicados. Esse saber conferido aos idosos está associado a dois conceitos de envelhecimento descritos por Gottlie (2007): o biológico normal, que abrange as mudanças inevitáveis e comuns a todos os idosos, decorrentes do processo de envelhecimento; e o biológico usual, que associa essas alterações fisiológicas à prevalência de doenças crônicas. Percebe-se, nos discursos dos longevos, um leque variado de situações vinculadas ao envelhecimento biológico. Entretanto, as doenças relacionadas ao envelhecimento usual mostram um apontamento importante, emergindo em possíveis preocupações dos longevos, não somente com o processo de envelhecimento, mas também com o adoecimento associado.

Os coeficientes referidos de doenças cardiovasculares ratificam essa associação entre a idade e adoecimento, vista por 38 (79,17\%) dos idosos do sexo feminino e 28 (80\%) do masculino, o que pode ser um reflexo da atuação da doença cardiovascular como uma das principais representantes do cenário usual de doenças associadas ao envelhecimento (GOTTLIE, 2007).

Não se pode deixar de descartar que esse saber provém da massificação de práticas educativas como um incremento de políticas públicas de saúde, sendo um facilitador na correlação do conhecimento informado aos fatores de riscos e do reconhecimento da doença pelos longevos.

Outras narrativas podem estar agregadas às tensões que circundam a capacidade de gerenciamento da autonomia e de realização do autocuidado pelo idoso, como é o caso das oftalmopatias, dos processos neurodegenerativos e da atrofia muscular, que não estão atribuídos, inicialmente, ao cenário educativo ou ao estado de adoecimento. 
Tabela 1. Doenças/problemas de saúde relacionados pelos idosos entrevistados

\begin{tabular}{lccrr}
\hline & Mulheres & \multicolumn{1}{c}{ Homens } \\
\hline Doença/Problemas & $\mathbf{N}$ & $\%$ & $\mathbf{N}$ & $\%$ \\
Perda de cabelo & 08 & 16,67 & 32 & 91,42 \\
Atrofia muscular & 12 & 25,00 & 01 & 2,86 \\
Hiperlipidemia & 06 & 12,50 & 19 & 54,29 \\
Hipogonadismo & 43 & 89,58 & 01 & 2,86 \\
Neurodegeneração & 14 & 29,17 & 01 & 2,86 \\
Alterações oftálmicas & 45 & 93,75 & 35 & 100,00 \\
Telangiectasias & 10 & 20,83 & 01 & 2,86 \\
Alterações cutâneas & 25 & 52,08 & 02 & 5,72 \\
Câncer & 10 & 20,83 & 04 & 11,44 \\
Doença vascular & 38 & 79,17 & 28 & 80,00 \\
Diabetes Mellitus & 18 & 37,50 & 21 & 60,00 \\
\hline
\end{tabular}

Fonte: Elaboração própria

Essas tensões, mediadas pelo grau de adoecimento ou por aspectos fisiológicos relacionados à idade, possuem um imaginário incapacitante e influenciam a mensuração da qualidade de vida pelos idosos, sendo, por sua vez, mais aplicados na autoavaliação da qualidade de vida pelos mesmos do que os fatores não médicos (SEIDL; ZANNON, 2004, P. 582).

A transmissão do conhecimento acerca dos processos patológicos, embora não seja objeto da atenção da promoção da saúde, colabora para a capacitação individual e coletiva, e traz reflexões significativas da visão do idoso sobre si mesmo, sua vulnerabilidade e a autonomia para desempenhar um papel na "prevenção dos fatores determinantes e/ ou condicionantes de doenças e agravos à saúde", conforme reza um dos objetivos específicos da Política Nacional de Promoção da Saúde (BRASIL, 2010, P. 18). Dessa forma, as condições de vida devem ser evidenciadas, juntamente à qualidade de vida, como cernes da promoção da saúde (MINAYO; HARTZ; BUSS, 2000).

As doenças ou os problemas de saúde mais descritos pelas longevas foram as alterações oftálmicas - 45 (93,75\%); o hipogonadismo - 43 (89,58\%); as doenças vasculares - 38 (79,17\%); e as alterações cutâneas - 25 (52,08\%). Assim como as mulheres, os homens apresentaram percentuais expressivos de alterações oftálmicas - 35 (100\%) e de doenças vasculares - 28 (80\%), como também perda de cabelo - 32 (91,42\%) e diabetes mellitus - 21 (60\%). Percebe-se a ocorrência de uma estratificação e uma hierarquização do conhecimento adquirido, atribuído ao gênero: as idosas possuem narrativas que dão ênfase ao conhecimento relacionado ao corpo e à incapacitação, enquanto os homens idosos demonstram conhecimento direcionado ao processo de adoecimento. Esses achados podem ser uma justificativa para o menor número de agendamento de consultas preventivas por parte dos homens, reiterado pelo entendimento de Alves et al. (2011), que afirma, em seus estudos, a negligenciação dos homens no tratamento preventivo e a busca do tratamento da enfermidade.

Com relação à pergunta referente ao conhecimento de longevos e longevas quanto à identificação de fatores de risco e agravos à saúde, os mesmos apresentaram narrativas unânimes sobre a aquisição desse saber, decorrentes das vivências individuais dentro do processo de envelhecimento e/ou 
adoecimento, ou pela transmissão de saberes por atividades educativas. Há uma hegemonia no discurso acerca da origem da informação, pautada no processo de envelhecimento para ambos os sexos, tendo destaque as ações educativas para as idosas.

Dentre os fatores de risco apontados, destacaram-se, para ambos os sexos: a alimentação inadequada para 46 (95,83\%) das longevas e para $30(85,71 \%)$ dos longevos; e o tabagismo para $16(33,33 \%)$ delas, bem como para $16(45,71 \%)$ deles. As desordens alimentares podem ser decorrentes da necessidade que a mulher apresenta de atender os padrões sociais de beleza. Já no caso do tabaco, o aumento do número de mulheres nas classes pobres, as dificuldades de acesso à educação e, paradoxalmente, a busca pela afirmação social, utilizando o status conferido ao tabaco, foram determinantes para esse processo (BORGES; BARBOSA, 2009). Não obstante, os fenômenos da feminilização - educacionais e da pauperização - agregam-se à tendência mundial de iniciação do uso de tabaco e de bebida alcoólica, através de estímulos midiáticos, que associam o tabaco à independência, à autoafirmação e à ascensão social (BORGES; BARBOSA, 2009). Apesar da descrição do tabagismo como fator de risco, ainda se faz necessário intensificar as ações de educação em saúde para a desconstrução dessas práticas, bem como a conscientização populacional acerca da correlação entre o tabagismo e a qualidade de vida.

Em uma avaliação de maior acurácia, nota-se que outros fatores de risco são evidenciados distintamente entre os sexos, emergindo nas influências de gênero dentro dos contextos sociais, históricos e culturais. As mulheres idosas compreendem o uso de bebidas alcoólicas como algo prejudicial à saúde, enquanto os homens não têm a mesma compreensão. O desenvolvimento do uso do álcool e suas implicações para as mulheres passam por distintos trajetos, quando comparados com os homens. No âmbito social, a mulher que utiliza o álcool é considerada imoral, do tipo que exerce práticas e comportamentos inadequados. Ambos os aspectos podem ser determinantes no apontamento acentuado do álcool, pelas mulheres, como fator de risco (RONZANI; FURTADO, 2010).

Os fatores de risco apontados como menos importantes foram: o estresse - $10(20,83 \%)$ e 1 ( $2,86 \%)$; e o sedentarismo - 14 (29,17\%) e $22(62,86 \%)$, mulheres e homens idosos, respectivamente. Esses achados, possivelmente decorrentes do grau de desvalorização atribuído a tais componentes, são decodificados como um padrão normativo dentro da sociedade moderna, tornando-se 'algo passível de convivência' no cotidiano.

Diante dessa gama de informações a serem desveladas, denota-se a relevância da construção e a utilização de métodos avaliativos sistemáticos capazes de aferir o grau de conhecimento do longevo e as relações feitas com a qualidade de vida, desenhando um processo educativo sensibilizado pelas diferentes nuances socioculturais e etárias, capazes de promover equanimidade informativa e instrumentalizar o idoso para o alcance do empowerment, fortalecendo e capacitando a população para a tomada de decisões e a busca de melhores condições de saúde (BUss, 2000). Todavia, apesar da incontestável importância dos instrumentos avaliativos da qualidade de vida, Seidl e Zannon (2004) percebem a relutância dos profissionais de saúde em utilizá-los na assistência aos usuários.

\section{Hábitos de vida do idoso}

A prática de atividades saudáveis é condicionante para o alcance da qualidade de vida, que, pela definição de WHOQOL (APUD SEIDL; ZANNON, 2004, P. 582), é algo subjetivo, interligado à "percepção da pessoa sobre o seu estado de saúde e sobre os aspectos não médicos do seu contexto de vida", sob uma ótica multidimensional. A percepção dos idosos possui caráter reducionista frente aos conceitos de práticas saudáveis e de qualidade de vida, 
não sendo mencionados outros fatores interferentes, tais como violência, desemprego, subemprego, falta de saneamento básico, condições habitacionais e crescimento urbano, dificuldade de acesso à educação, fome, poluição, qualidade do ar e da água consumida.

O conceito de promoção da saúde não pode ser alcançado através de ações intrassetoriais isoladas, pois pertence a um projeto maior, de uma sociedade solidária. Permeia a unção de diversos saberes e práticas "multi e intersetorial" sobre os problemas de saúde iminentes (BUSS, 2001, P. 174). Essas práticas assistenciais devem caminhar paralelas à melhoria das condições sanitárias e habitacionais, à garantia do acesso a serviços, saúde e meio ambiente. Assim sendo, a integração e a consolidação de estratégias transversais nos diversos segmentos sociais torna-se indispensável para a construção de uma qualidade de vida, reduzi$\mathrm{da}$, atualmente, a consultas assistencialistas e processos educativos.

A alimentação foi o quesito mais apontado por 42 (87,5\%) mulheres e por $27(77,14 \%)$ homens, preponderantemente, como prática saudável. No entanto, apesar de grande parte desses idosos considerar a alimentação como uma ferramenta essencial para o alcance de hábitos saudáveis, apenas um grupo reduzido de mulheres - 34 (70,83\%) - e de homens - $9(25,71 \%)$ - referem ingerir diariamente uma dieta saudável, indicada pelo serviço de nutrição da unidade de saúde.

As dificuldades encontradas na execução de uma alimentação saudável podem ser dadas à ausência de ações específicas de setores intra e intersetoriais para a garantia do acesso à alimentação, levando-se em consideração as particularidades culturais, regionais e locais. Faz-se necessária, também, a reorientação dos serviços de saúde para a prevenção e o controle de agravos e doenças decorrentes da má alimentação, bem como a mobilização e a capacitação profissional para a educação em saúde, além da incorporação dos idosos no Sistema de Vigilância Alimentar e Nutricional habilitado, com vistas ao desenvolvimento de ações para a promoção da alimentação saudável (BRASIL, 2010).

Os dados acerca da alimentação saudável reforçam a ideia de distanciamento entre o conhecimento adquirido e o conhecimento executado, sendo fundamental a massificação de ambos por meio da educação em saúde, a fim de proporcionar aderência a essa prática saudável. Embora a atividade física tenha sido menos referida pelos idosos, as narrativas relatam o seguinte panorama: $42(87,5 \%)$ idosas e $21(60 \%)$ idosos realizam tais atividades, sejam elas externas (academia ou caminhadas); oferecidas na unidade de saúde - 37 (77,08\%) e 12 $(34,29 \%)$ longevas e longevos, respectivamente; ou em parceria com programas públicos de saúde - 26 (54,17\%) e 19 (54,28\%) delas e deles, também respectivamente.

A oferta da atividade física pela UBS denota uma tentativa oportuna de garantir a efetividade da promoção da saúde ao idoso e pode ser considerada como uma grande estratégia observada pela significativa aderência dos mesmos, o que amplia as relações sociais e as oportunidades para praticá-las. Ademais, essa proposta oferecida, de prática desportiva, não é obstaculizada por questões de cunho financeiro, sendo acessível a qualquer idoso, independentemente da idade e da condição socioeconômica, e isto promove a integração e a socialização grupal (GOBBI ET AL., 2008).

Desses idosos, 34 (70,83\%) mulheres e $13(37,14 \%)$ homens relacionaram a prática de atividade física à busca de um estilo de vida ativo e à manutenção da capacidade funcional e da autonomia, contrapondo o que Gobbi et al. (2008) definem como um dos principais entraves para a prática de exercícios: o estado de envelhecimento, o sedentarismo e a existência de problemas de saúde.

As demais ações específicas de promoção da saúde como prevenção e controle do tabagismo, a redução da morbimortalidade 
pelo uso de álcool, outras drogas e acidentes de trânsito e o estímulo à cultura de paz são mencionados pelos idosos somente como conteúdos essencialmente conceituais, dentro das atividades educacionais em saúde, e não como exercícios de práticas preventivas e promocionais propriamente ditas.

\section{Educação, participação social e empoderamento}

A existência da educação dentro das unidades de saúde e a participação dos entrevistados em atividades educativas foram relatadas por 48 (100\%) longevas e 32 (91,42\%) longevos, o que mostra um possível reconhecimento do processo educativo como um dispositivo viabilizador da promoção da saúde e de mudanças paradigmáticas, concomitante a profissionais de saúde e idosos, norteando as ações intersetoriais e os próprios longevos, restritos, até pouco tempo atrás, a uma condição de passividade.

O perfil de atividades educativas implantadas apresentou um discurso homogêneo nas narrativas dos idosos, no que tange à disponibilização e à ocorrência do citado dispositivo, de métodos de aprendizagem e dos conteúdos ministrados dentro desse processo. Dos 83 idosos cadastrados na unidade, os totais discriminados em seguida referenciaram, em maior escala, as palestras, os grupos de apoio e a orientação durante as consultas: $69(83,13 \%), 43(51,87 \%)$ e $12(14,46 \%)$, respectivamente.

As palestras ministradas, citadas pelos idosos, aconteciam aleatoriamente, durante o aguardo das consultas ou anteriormente agendadas, e apresentavam conteúdos fixos, previamente selecionados, direcionados ao conceito, ao tratamento e à prevenção de doenças crônicas não transmissíveis - 41 (49,40\%); aos hábitos alimentares - 20 (24,10\%); ao tabagismo - 7 (8,43\%); e ao etilismo - 3 (3,61\%).

Os idosos informaram que os grupos de apoio aconteciam quinzenalmente, oportunizando temas inseridos dentro da pauta de promoção da saúde: cuidados com o corpo - 21 (25,30\%); alimentação saudável - 20 (24,09\%); prática corporal/atividade física - 20 (24,09\%); prevenção e controle do tabagismo - 24 (28,91\%); abusivo de álcool - 29 (34,94\%); e drogas - 5 (6,02\%). Esses componentes educativos foram primariamente relacionados aos riscos comportamentais passíveis de mudanças, que estariam, pelo menos em parte, sob o controle dos próprios indivíduos (BUss, 2000).

As narrativas dos entrevistados mostraram a necessidade de se utilizar a educação em saúde nas consultas, em caráter esclarecedor e conscientizador, ofertando orientações específicas sobre as situações advindas do envelhecimento - 6 (7,23\%) - ou de adoecimento - $9(10,84 \%)$ - vivenciadas pelos idosos naquele momento. A promoção da saúde pouco é aplicada nesse estágio, pois o interesse está atrelado a um modelo curativo e não à garantia da autonomia e do autocuidado, dentro de um modelo promocional de saúde.

Segundo os entrevistados, não foi realizada uma investigação para avaliar o grau de aprendizado dos mesmos em relação aos conteúdos abordados. Ademais, não são referidas possíveis discussões sobre os conceitos de prevenção de acidente de trânsito, violência e estímulo à cultura e desenvolvimento sustentável, o que mostra a necessidade de uma capacitação profissional e de métodos avaliativos periódicos do processo educacional, conforme rezam as especificações das diretrizes operacionais da educação em saúde (BRASIL, 2007).

Foram referidos dois métodos de ensino-aprendizagem na abordagem dos temas: a educação em saúde tradicional, com ação verticalizada e centrada no processo saúde-doença, utilizada nas palestras e nos consultórios; e a educação em saúde dialógica, focada nas ações primárias, na busca crítico-reflexiva das condições de vida, possibilidades e estratégias de mudanças. Este último método foi massificadamente aplicado nos grupos de apoio, priorizando a construção e a horizontalização do conhecimento, o 
desenvolvimento de habilidades pessoais e sociais, a sustentabilidade grupal, a autonomia e o empoderamento do idoso.

Observa-se a utilização, nesses processos, do 'conhecimento-regulação' e do 'conhecimento-emancipação' como métodos educativos (LOPES; TOCANTINS, 2012; SANTOS, 2001). Embora o primeiro seja utilizado para a transmissão da informação como fato e elemento de ordem sobre as coisas e os outros, o "conhecimento-emancipação" tem tido maior aderência por parte dos idosos, pois os torna mais participativos dentro do processo educativo (SANTOS, 2001).

Percebe-se que parte das estratégias educativas aplicadas busca a autonomia do idoso, pautada na aquisição de autorrespeito, autoconfiança e autoestima, estes que estão, por sua vez, conectados às redes de reconhecimento social. Sem elas, o longevo não dispõe de elementos suficientes para enfrentar as situações de vulnerabilidade derivadas de um contexto social que tende a desvalorizar seu modo de vida (SCHUMACHER, 2013).

O grupo de apoio mostrou-se como o método mais eficiente na transmissão de conhecimento, não só pelo maior número de temas descritos, mas por agregar conhecimento, informação e emancipação, direcionando esses itens a conteúdos pré-elaborados e ao atendimento dos diferentes graus de dificuldades individuais. A estes graus estão vinculados os prejuízos e ganhos compartilhados com o grupo, o que despertou, entre os idosos, a formação de consciência crítica sobre a realidade social, sobre si próprios, e construiu novas habilidades e atitudes em benefício de sua saúde e sua promoção, em favor de suas vidas cotidianas e do seu desenvolvimento pessoal e coletivo. É no trabalho em grupo com pessoas idosas que se permite a articulação e o debate das questões peculiares a uma determinada população, como saúde, qualidade de vida, interação social, autoestima e vulnerabilidade (LOPES; TOCANTINS, 2012; BRASIL, 2006).

Dessa forma, a atividade grupal pode ser considerada como um processo educativo atento às diferenças socioculturais da população, não se limitando às informações cedidas pelos profissionais como verdades únicas e absolutas (BRASIL, 2007). Quando transportamos os mais variados estágios de consciência para a complexidade da educação em saúde, constatamos que a consciência em saúde é mediada pelo processo sequencial de ação-reflexão-ação e possibilitada pelo diálogo, que predispõe o rompimento da cultura do silêncio e desperta os usuários para novas análises acerca da sua saúde (ALVIM; FERREIRA, 2007).

Todavia, apesar dos relatos da existência de orientação acerca de hábitos saudáveis, de cuidados com a saúde e de autocuidado, os conteúdos proferidos na educação em saúde, independentemente do método escolhido, devem ser ampliados, juntamente com a diversificação e o aprofundamento dos conteúdos pré-existentes, haja vista que o conhecimento que os entrevistados afirmam ter, para identificar fatores de risco, não fornece elementos suficientes para o empoderamento pleno e a construção de habilidades, assim como para o desenvolvimento da autonomia e, consequentemente, do autocuidado.

Com relação ao conhecimento acerca de doenças/problemas provenientes do envelhecimento, as longevas - 47 (97,91\%) - se mostraram mais informadas dos que os longevos - 29 $(82,85 \%)$. Esse diferencial biológico atribuído ao gênero constitui um importante marcador social e deve ser valorizado no momento da elaboração de métodos de atuação e na abordagem ao longevo, com vistas à minimização das diferenças dicotômicas e à oportunização de condições equânimes de saúde.

\section{Conclusão}

A promoção da saúde consiste em uma nova modalidade conceitual e prática de políticas públicas, visando ao indivíduo e ao coletivo, através da busca de qualidade de vida, autonomia e estímulo ao autocuidado. 
Percebe-se que a promoção da saúde se expressa fundamentalmente nas unidades básicas através da educação em saúde, presente nas práticas desenvolvidas pelos profissionais envolvidos.

A educação em saúde, embora possua métodos e segmentos distintos, não se limita apenas a transmitir conhecimento à comunidade, mas estabelece vínculos entre assistidos e profissionais, e promove a participação ativa da comunidade, a inclusão social e constantes remodelagens conceituais destes indivíduos, quanto a hábitos que comprometam a saúde e a qualidade de vida daquela população. No entanto, existem fortes marcadores que delimitam e obstaculizam as práticas educativas e de promoção da saúde, como, por exemplo, o caso das questões de gênero, que necessitam de uma reorganização de

\section{Referências}

ALVES, R. et al. Gênero e saúde: o cuidar do homem em debate. Revista Psicologia: teoria e prática, São Paulo, v. 13, n. 3, 2011, p. 152-166.

ALVIM, N. A. T.; FERREIRA, M. A. Perspectiva problematizadora da educação popular em saúde e a enfermagem. Texto Contexto - enferm., v. 16, n. 2, 2007.

BORGES, M. T. T.; BARBOSA, R. H. S. As marcas de gênero no fumar feminino: uma aproximação sociológica do tabagismo em mulheres. Ciênc. saúde colet., Rio de Janeiro, v. 14, n. 4, ago. 2009. Disponível em: <http://www.scielo.br/scielo.php?script=sci arttext\&pid=S1413-81232009000400019\&lng=en\&nrm =iso $>$. Acesso em: 6 abr. 2014.

BRASIL. Fundação Nacional de Saúde. Diretrizes de educação em saúde visando à promoção da saúde: documento base - documento I/Fundação Nacional de Saúde. Brasília, DF: Fundação Nacional de Saúde, 2007. práticas a fim de minimizar as assimetrias e oportunizar condições equânimes de saúde a todos os idosos.

Embora a transmissão de conhecimento se faça presente, ela não é homogênea, o que torna necessária a intensificação das bases das políticas de promoção da saúde, incluindo métodos de avaliação do conhecimento oriundo do processo educativo, de detecção de possíveis falhas para a elaboração de estratégias de reversão, e de absorção dessas informações pelo idoso.

Pode-se contar com a educação em saúde como um instrumento promocional e de estímulo ao autocuidado, ponderando-se as informações fornecidas. Diante disso, entende-se que a educação e a promoção da saúde caminham juntas, gerando possibilidades para que o idoso se conscientize e empodere, objetivando sua qualidade de vida.
Ministério da Saúde. Política Nacional de Atenção Integral à Saúde do Homem: princípios e diretrizes. Brasília, DF: Ministério da Saúde, 2008.

Ministério da Saúde. Secretaria de Vigilância em Saúde. Secretaria de Atenção à Saúde. Política Nacional de Promoção da Saúde/Ministério da Saúde, Secretaria de Vigilância em Saúde, Secretaria de Atenção à Saúde. 3. ed., Brasília, DF: Ministério da Saúde, 2010.

BUSS, P. M. Promoção da saúde e qualidade de vida. Ciênc. saúde colet., Rio de Janeiro, v. 5, n. 1, 2000.

CARRARA, S. et al. A construção do conhecimento em gênero e sexualidade: histórias e perspectivas. Curso de especialização em gênero e sexualidade. Rio de Janeiro: CEPESC, 2010.

ENRIA, G.; STAFFOLANI, C. Contradicciones de los discursos que dificultan la transformación de las 
prácticas de promoción a la salud. Hacia la promoción de la salud, Calle, v. 15, n. 1, 2010.

FIGUEIREDO, W. Assistência à saúde dos homens: um desafio para os serviços de atenção primária. Ciênc. Saúde Colet., Rio de Janeiro, v. 10, n. 1, mar. 2005.

GOBBI, S. et al. Comportamento e Barreiras: Atividade Física em Idosos Institucionalizados. Psicologia: Teoria e Pesquisa, Brasília, DF, v. 24, n. 4, 2008, p. 451-458.

GOTTLIE, M. G. V. et al. Aspectos genéticos do envelhecimento e doenças associadas: uma complexa rede de interações entre genes e ambiente. Rev. Bras. Geriatr Gerontol., Rio de Janeiro, v. 10, n. 3, 2007.

KORIN, D. Novas perspectivas de gênero em saúde. Adolescência Latinoamericana, Porto Alegre, v. 2, n. 2, mar. 2001. Disponível em: <http://raladolec.bvs.br/ scielo>. Acesso em: 9 abr. 2010.

LOPES, M. S. V.; SARAIVA, K. R. O.; XIMENES, L. B. Analise do conceito de promoção da saúde. Texto Contexto Enferm., Florianópolis, v. 19, n. 3, jul./set. 2010, p. 461-468.

LOPES, R.; TOCANTINS, F. R. Promoção da Saúde e a Educação Crítica. Interface (Botucatu), Botucatu, v. 16, n. 40, mar. 2012. Disponível em: <http://www. scielo.br/scielo.php?script=sci_arttext\&pid=S1414$-32832012000100018 \& \operatorname{lng}=$ en $\& n r m=i s o>$. Acesso em: 11 jun. 2013.
MINAYO, M. C. S.; HARTZ, Z. M. A.; BUSS, P. M. Qualidade de vida e saúde: um debate necessário. Ciênc. saúde colet., Rio de Janeiro, v. 5, n. 1, 2000. Disponível em: <http://www.scielo. br/scielo.php?script=sci_arttext\&pid=S1413$-81232000000100002 \& \operatorname{lng}=\mathrm{en} \& \mathrm{nrm}=\mathrm{iso}>$. Acesso em: 9 dez. 2014.

RONZANI, T. M.; FURTADO, E. F. Estigma social sobre o uso de álcool. J. bras. psiquiatr., Rio de Janeiro, v. 59, n. 4, 2010 .

SANTOS, B. S. Da ciência moderna ao novo senso comum: a ciência, o direito e a política na transição paradigmática - a crítica da razão indolente: contra o desperdício da experiência. 3. ed. São Paulo: Cortez, 2001.

SCHUMACHER, A. A.; PUTTINI, R. F.; NOJIMOTO,

T. Vulnerabilidade, reconhecimento e saúde da pessoa idosa: autonomia intersubjetiva e justiça social. Saúde debate, Rio de Janeiro, v. 37, n. 97, jun. 2013.

SEIDL, E. M. F; ZANNON, C. M. L. C. Qualidade de vida e saúde: aspectos conceituais e metodológicos. Cad. Saúde Pública, Rio de Janeiro, v. 20, n. 2, abr. 2004. Disponível em: <http://www.scielo.br/scielo. php?script=sci_arttext\&pid=S0102-311X20040002000 27\&lng=en\&nrm=iso>. Acesso em: 9 dez. 2014.

\footnotetext{
Recebido para publicação em abril de 2014

Versão final em fevereiro de 2015

Conflito de interesse: inexistente

Suporte financeiro: não houve
} 